\title{
An Electronic Circuit for Measuring the Displacement of Pressure-Sensitive Diaphragms
}

\author{
Maurice L. Greenough and William E. Williams
}

\begin{abstract}
An instrument has been developed for indicating the position of thin pressure-sensitive diaphragms. As no appreciable load is placed on the diaphragm, the system is well adapted to the measurement of low absolute or differential pressures in the range from zero to 100 microns. The electronic instrument gives full-scale indication for motions of less than $5 \times 10^{-4}$ inch. Five tubes are incorporated in a circuit based upon the principle of the mutualinductance micrometer for the detection of diaphragm motion. The same instrumentation may be utilized in other applications requiring the indication of very small displacements.
\end{abstract}

\section{Introduction}

An electrical distance-measuring system designed for application to sensitive diaphragms has been successfully employed for pressure measurements in mass spectrometers. ${ }^{1}$ The same system is also applicable to many other problems requiring the remote indication of relative position. Specifically, the instrument described is well suited for the measurement of distances of $5 \times 10^{-4}$ in. as a full-scale value. Motions of less than $10^{-5}$ in. may be detected. Displacements of $0.05 \mathrm{in}$. may be indicated by suitable designs incorporating the same method of distance measurement.

The instrument, coupled to a pressure-sensitive diaphragm, is shown in figure 1. With the exception of the distance-measuring element itself, all of the electronic circuit is housed in the steel cabinet. The pressure cell and distance-measuring element may be seen inside the glass bell jar. In operation the bell jar is evacuated, and the measured pressure is that inside the cell. The cell is connected to the mass spectrometer by an inlet through the bottom of the glass bell. In other applications it may be preferable to interchange the unknown pressure and the vacuum. Instrument performance would not be affected.

1 Vernon H. Dibeler and Fidel Cordero, A diaphragm-type micromanometer for use on a mass spectrometer, J. Research NBS 46, 1 (1951) RP 2167.

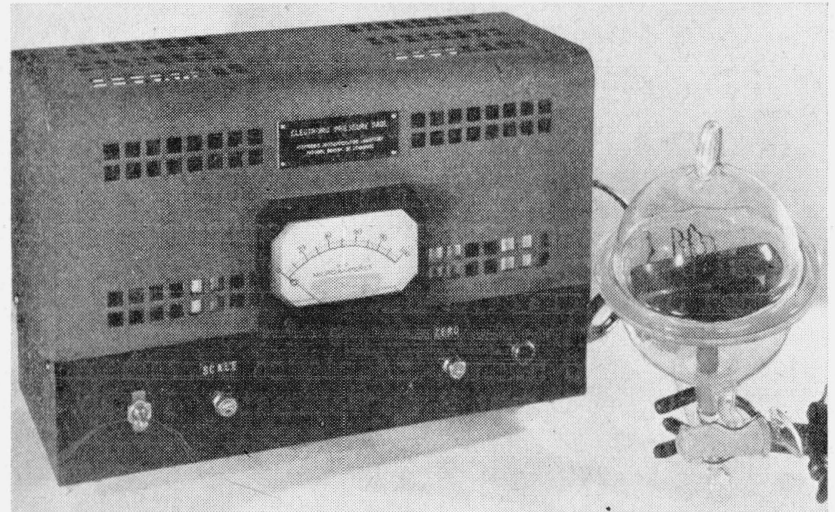

FIGURE 1. Electrical micromanometer system, showing instrument, evacuated glass bell, and pressure cell.
The distance-measuring element used for observing motion of the pressure-responsive diaphragm is of the mutual-inductance type. ${ }^{2}$ Two air-core coils are wound on a single form that is mounted in proximity to the diaphragm. The presence of the metal diaphragm adjacent to the coils produces partial shielding between them, and motion of the diaphragm as a result of pressure changes causes a variation in the mutual inductance between the windings. Suitable electronic circuitry is then utilized to provide the desired pressure indication.

The actual diaphragm motion is approximately $0.0005 \mathrm{in.}$ (500 microinches) for full-scale indication on the most sensitive meter range. For this motion, the mutual inductance is changed 1.2 percent. W hile at first glance it would appear that other and more sensitive distance-measuring systems could be employed, the ruggedness and adaptability of the present system give it advantages for this application. It would not be difficult, for example, to obtain a 10-percent change in capacitance between two plates at reasonable spacing. However, this initially greater percentage change would be masked by the capacitance of the interconnecting cable, or would require that part of the circuitry be mounted in close proximity to the pressure cell. Rather critical diaphragm alinement would also be required, as both initial clearance and tilt affect the magnitude of capacitance variation. Magnetic core type distancemeasuring elements have the disadvantage of reacting on the diaphragm, as well as requiring magnetic material attached to the diaphragm.

The mutual inductance micrometer has advantages in this application in that it possesses linear response as a function of displacement and does not appre. ciably load, or alter the characteristics of, the diaphragm. As the distance-measuring element exhibits low-impedance, cable length and resulting capacitance is not critical. The device requires a reasonably flat area on the diaphragm approximately $5 / 8$ in. in diameter, of a nonmagnetic, low-resistance metal at least 0.0005 in. thick. These conditions were satisfied for this application by fabricating the entire diaphragm in a suitable shape of 0.001-in. brass sheet. (Construction of the pressure cell is described in the paper referred to in footnote 1).

\footnotetext{
${ }_{2}^{2}$ Technical details of electronic micrometer, Electronies 20, 172 (Nov. 1947).
} 


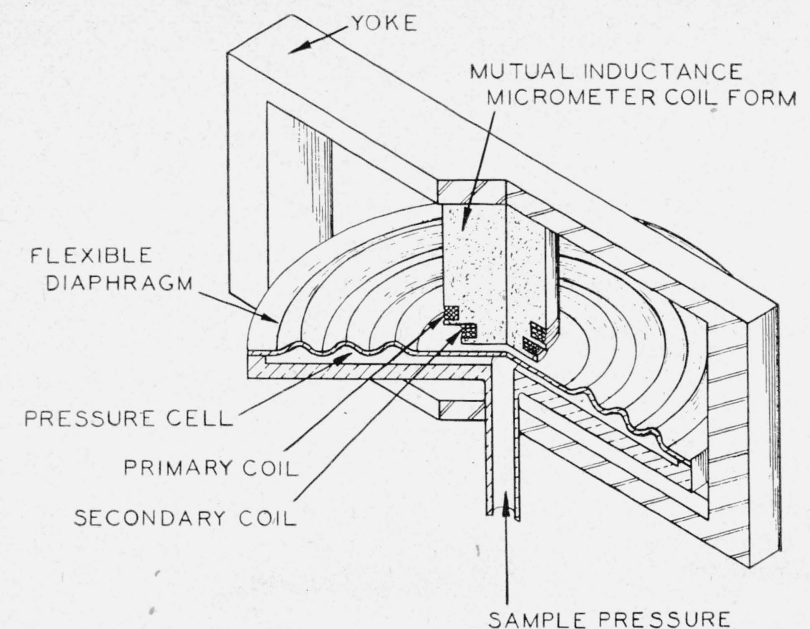

Figure 2. Cut-away sketch of pressure cell and mutual inductance micrometer.

The relative locations of the pressure cell and micrometer windings are indicated in figure 2. A stainless-steel yoke provides the mounting to hold the micrometer coil form and the pressure cell in the proper relation. The flexible diaphragm of the cell is nearest to the face of the supporting form of the distance-measuring element, or probe. As the pressure being measured is that inside the cell, zero absolute pressure causes the diaphragm to assume its most remote position with respect to the probe windings. Increased pressure then produces expansion of the cell and consequent reduction in spacing between diaphragm and windings.

Normally, the initial clearance for zero pressure is set at about 0.005 in. between the diaphragm and the end of the probe. A pressure of $250 \mu$ is sufficient to close the gap. The diaphragm moves approximately 20 microinches per micron of mercury pressure.

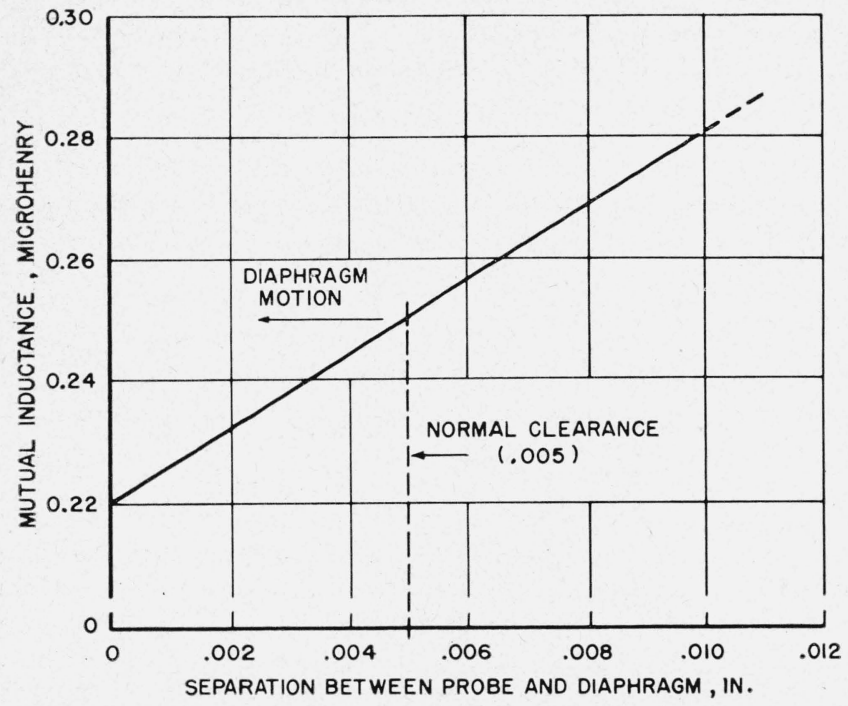

FIGURE 3. Electrical characteristics of mutual inductance micrometer for measuring diaphragm position.
The electrical characteristics of the mutual inductance probe are shown in figure 3 . When the metal surface is in contact with the end of the probe, the coupling between the two windings is at a minimum, because eddy currents induced in the metallic surface set up an opposing electromagnetic field, whose cancellation effect is then greatest. Coupling between the windings increases with the spacing between probe and metal surface. The relation has the form of an initial or minimum coupling to which is added a component proportional to separation. By suitable design, the proportionality of this component is made linear to within 1 percent over a range of separation from 0 to 0.02 in. As the motion. of the diaphragm is restricted to a few thousandths of an inch, the wide range of linear response is more than adequate. However, it allows variation in the initial position of the diaphragm without altering the sensitivity of the instrument, as the change of mutual inductance for a given incremental motion is constant. Initial clearance is set at the value of 0.005 in. to provide some tolerance for misalinement.

The cutaway view of the probe in figure 2 illustrates the approximate geometry of the probe windings. The primary winding is usually placed in the outer slot, which is further away from the metal surface, whereas the secondary is located in the closer one. The secondary is wound with several times the number of turns on the primary. As its resistance is not critical, fine wire is used on the secondary in order to accommodate the winding in as narrow a slot as possible. The narrow slot and thin supporting wall are desirable in that mounting of the windings closer to the diaphragm is possible. In the design of the probe used in this equipment, the minimum value of coupling as set by these factors is equivalent to about 0.035 in. The desirability of a low value of minimum coupling is that less precision is then demanded of the circuits provided for its cancellation. However, the actual change in mutual inductance in microhenrys (the slope of the relation in fig. 3), is substantially unaffected by changes in minimum coupling.

Details of the probe and its windings are given in the appendix, part $\mathrm{A}$.

\section{Circuit Details}

The circuit diagram is shown in figure 4 , and component specifications are listed in the appendix, part B. The source of radio-frequency power is a $6 \mathrm{~V} 6$ oscillator arranged for two-terminal operation by means of a nonresonant feedback transformer, $L_{5}$ and $L_{6}$. The frequency of oscillation is determined by reasonance of the shunt-fed tank circuit formed by $C_{2}$ in combination with the total inductance of the primary windings of the current transformer, $L_{3}$, and mutual-inductance micrometer, $L_{1}$. The oscillator output is approximately 1 amp. at a frequency of $2.5 \mathrm{Mcs}$.

The current transformer serves several purposes. Its chief function is to provide a means of obtaining a voltage that is proportional to the current in the mutual-inductance micrometer. It is also necessary 


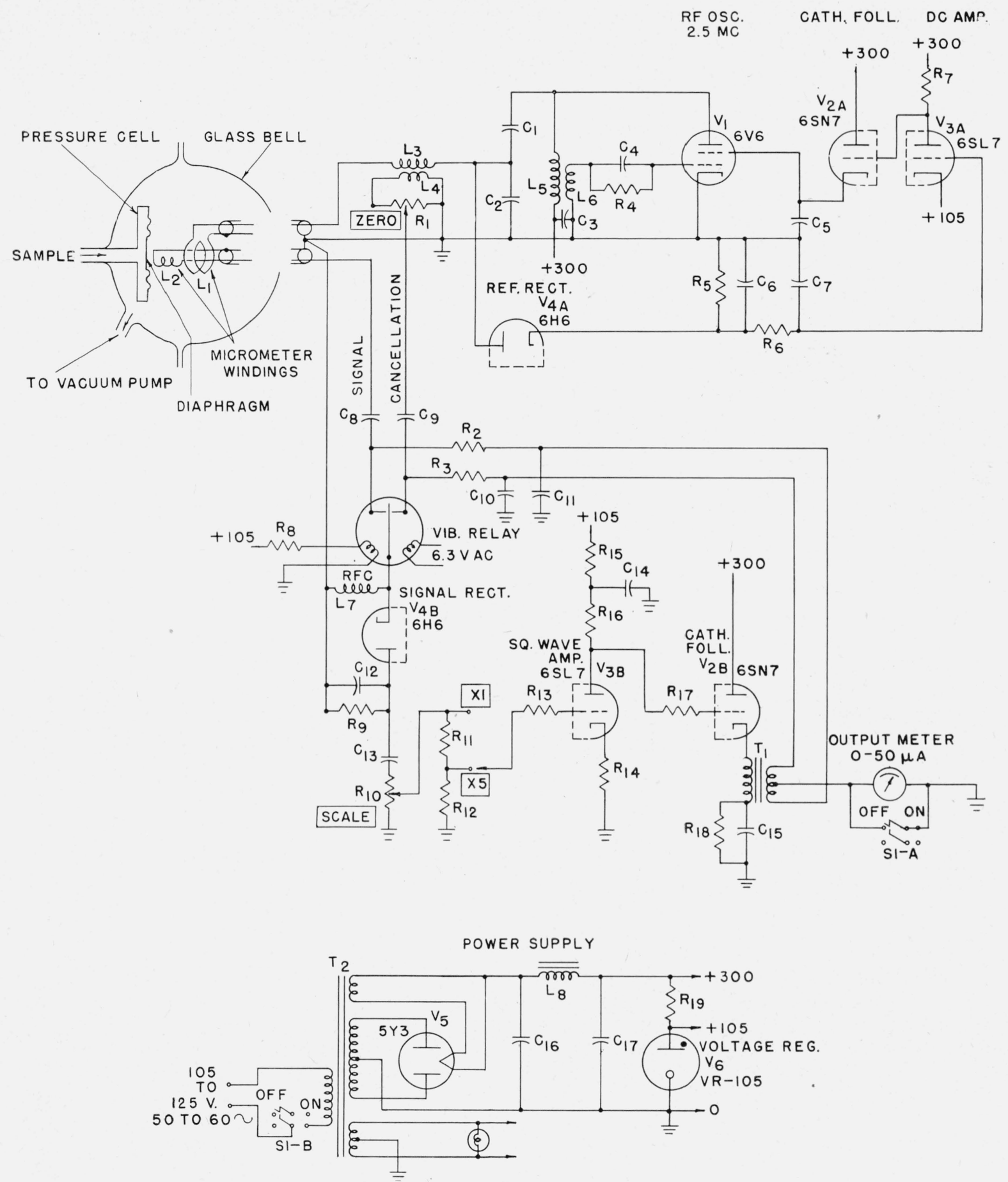

Figure 4. Circuit diagram of electrical micromanometer. Values of components are listed in the appendix, part B. 
for limiting the power input to the distance-measuring element to avoid inductive heating of the diaphragm. Only about 10 percent of the voltage across $C_{2}$ appears across the primary winding of the micrometer element.

To obtain constancy of output indication for a given pressure, it is necessary to regulate the amplitude of the radio-frequency current in the micrometer primary. It is accomplished in this instrument by regulating the oscillator output voltage, depending upon the stability of the primary inductance of the current transformer, probe, and cable to secure constant current. Frequently shift, arising from changes in the value of the tuning capacitor for example, produces a corrective variation in current, so that the output of the mutual-inductance micrometer remains unchanged.

The regular circuit commences with a $6 \mathrm{H} 6$ halfwave peak rectifier, $V_{4 A}$, the output of which is fed to the grid of a $6 \mathrm{SL} 7$ direct-current amplifier, $V_{3 A}$. A fixed reference voltage from a gaseous regulator tube, $V_{6}$, is applied to the cathode of the 6SL7. The difference between these two is the grid bias on the tube. The resulting plate voltage on the tube is transmitted through a $6 \mathrm{SN} 7$ cathode follower, $V_{2 A}$, to control the screen potential on the $6 \mathrm{~V} 6$ oscillator, completing the regulating loop. The amplitude of oscillation is thereby automatically held at about $102-v$. peak. The loop gain of the regulator circuit is approximately 70 , so that the effect of varying tube characteristics, line voltage, etc. is greatly reduced.

In order to obtain a stable source of voltage for cancellation of the coupling at normal clearance, a secondary winding is placed on the current transformer. 'Through a potentiometer, $R_{1}$, an adjustable $\mathrm{r}-\mathrm{f}$ voltage source is obtained. Voltage from this source bears the same proportional relation to oscillator voltage and frequency as that from the secondary of the mutual inductance micrometer. This potentiometer provides adjustment for equality of current transformer and micrometer outputs, under conditions of zero differential pressure on the diaphragm. Thereafter, only relative geometrical changes can disturb the balance between these two voltages. Changes in inductance of the interconnecting cables for example, can cause only identical effects in the two secondaries but no unbalance. The result is an extremely stable zero indication.

The absolute magnitude of these voltages is proportional to the exciting current and frequency. As mentioned, the factors of exciting current and frequency are fixed by virtue of the constant-voltage regulator circuit in conjunction with stable inductances. Cable inductance increases the impedance of the inductive branch of the tank circuit and lowers the frequency tending to reduce current flow in that branch. Change in lead inductance therefore can produce similar variations in indicated output. However, lead inductance itself is small compared to that of the current transformer, so that minor variations are negligible. Had variations from this cause been found appreciable, a step-up type of current transformer feeding the $6 \mathrm{H} 6$ rectifier could have been employed. For this instrument, the additional complexity was not felt warranted, as scale-factor stability was found to be good with the regulating circuit employed.

When the primary winding of the probe is excited with high-frequency current, the output of the secondary is a voltage of the same frequency, whose magnitude is proportional to the mutual inductance. The secondary voltage is fed to one contact of a single-pole-double-throw vibrating relay actuated at 60 cycles. The other contact is connected to the cancellation voltage of the same frequency from $R_{1}$. The resultant voltage at the arm of the vibrating relay becomes a square-wave, amplitude-modulated carrier, whose modulation is proportional to diaphragm excursion. Zero modulation is made to correspond to zero differential pressure by adjustment of $R_{1}$. The modulated carrier is rectified, resulting in a 60-cycle square-wave signal. This is amplified and fed back through radio-frequency isolation circuits to the contacts on the vibrating relay. The synchronous rectification so obtained provides current for actuation of a direct-current microammeter.

The relay used was a 60 -cycle vibrating one with mercurv-wetted contacts. A steady direct-current from $\dot{V}_{6}$ through $R_{8}$ is put through one of its windings to bring the armature to the central position. The addition of 60-cycle alternatingcurrent in the other winding causes equal dwell time on each of the contacts, producing symmetrical square-wave modulation. In practice, the magnitude of $R_{8}$ is chosen to provide equal dwell time.

The square-wave component of rectifier output ${ }^{3}$ is fed through an attenuator, $R_{10}$, for scale factor adjustment during calibration. Two scale ranges are provided with a 5:1 ratio $\left(R_{11}\right.$ and $\left.R_{12}\right)$.

The square-wave is next passed through an amplifier stage, whose gain is stabilized with inverse feedback by omitting the cathode bypass capacitor on $R_{14}$. Following the amplifier is a direct-coupled cathode follower that feeds an output transformer of about 2.5:1 stepdown to the center-tapped secondary. The square-wave voltage at the secondary is connected through the low-pass filter, $R_{2}$, $R_{3}, C_{10}$, and $C_{11}$ to the same relay contacts that handle the carrier frequency signals. The purpose of the filter is to prevent loading of the carrier signal sources by the output transformer. Blocking capacitors $\dot{C}_{8}$ and $C_{9}$ prevent loading of $T_{1}$ by the radiofrequency circuits. As resistances are required by amplifier loading considerations, and should preferably be precision components, the use of wirewound elements as $\mathrm{r}-\mathrm{f}$ chokes served both functions. No interference was observed between the low and high frequency circuits with the component values shown. The only critical point is that the radiofrequency choke from relay arm to ground have low 60-cycle impedance to avoid spurious feedback.

${ }^{3}$ Vibrating relays in this service were sometimes found to display large switch ing transients. Some methods of transient reduction are discussed in the appendix, part C. The particular one used was satisfactory in this respect. 


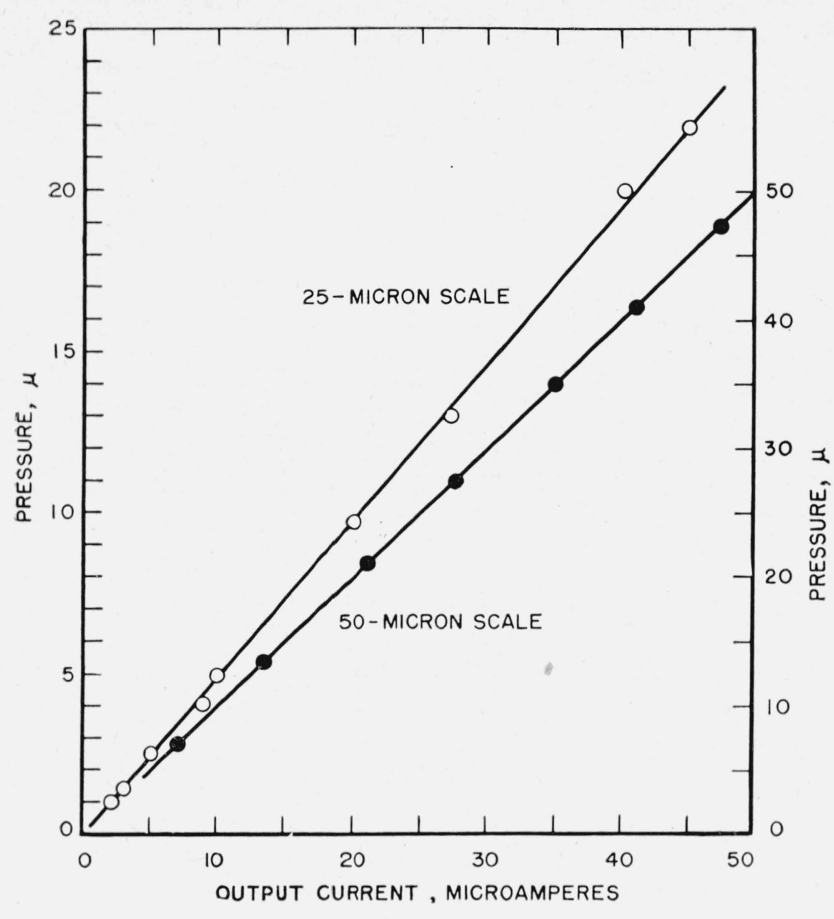

Figure 5. Output current and pressure relationship.

Solid points refer to the right-hand scale. The low-current portion of the range, shown in expanded form with the open circles and the left-hand scale, was produced by adjustment of the scale control.

The reflex connection acts as its own synchronous rectifier, saving the use of a separate detector tube.

\section{Performance}

The over-all response of the system is shown in figure 5, where the relationship between output current and gas pressure is plotted. As the relation is a direct proportionality, calibration at only two points, for intercept and slope, is required. Intercept adjustment, at zero pressure, is controlled by the zero potentiometer, $R_{1}$, and is set so that zero output current is indicated for a perfect vacuum or zero differential pressure. The slope adjustment for magnitude of indication for known gas pressures is determined by the scale control, $R_{10}$. Typical resultant curves for output current are given in figure 5, reproduced from the paper referred to in footnote 1 .

The stability of the instrument in the vicinity of zero pressure indication is good. As a result of the circuits employed for obtaining cancellation voltage, there is little of an electrical nature that can cause zero shift of serious magnitude. Obviously, the potentiometer used for adjustment of cancellation voltage must be stable. Line voltage changes of \pm 10 produce error readings of about $0.5 \mu$, in the form of a slow drift believed due to heater voltage effects. The cause of the variation observed is probably the dependence of the effective resistance of the $6 \mathrm{H} 6$ signal rectifier, $V_{4 B}$, upon heater voltage. As the sources of cancellation and micrometer signal voltages are not of equal impedance, there may be a

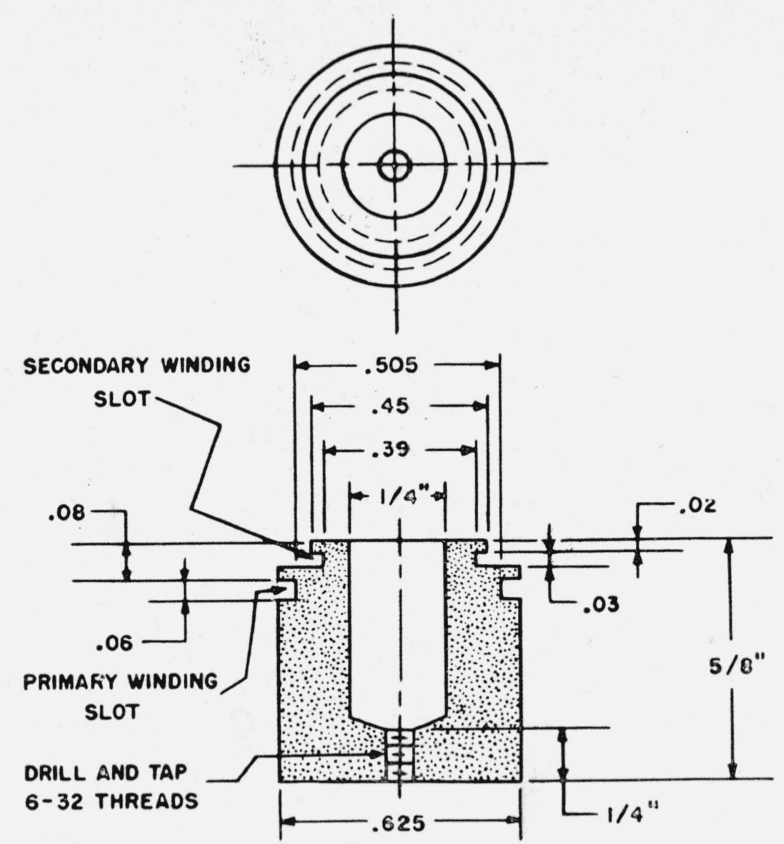

Figure 6. Construction details of probe.

differential change in rectification efficiency in the half-wave, peak circuit employed. For this specific application, the magnitude of drift encountered was considered negligible. Hum pickup in the amplifier can also give rise to zero shift with line voltage variations, inasmuch as the pickup is usually proportional to line voltage. Proper wiring and shielding will reduce any effects from this cause.

The stability of scale factor is also good, because of the high gain of the oscillator regulator circuit, and the use of inverse feedback and regulated plate potentials on the square-wave amplifier. Line voltage variations of $10 \mathrm{v}$ result in approximately 1-percent indicated pressure errors.

\section{Appendix}

A. Drawings showing the constructional and winding details of the mutual-inductance micrometer are presented ir. figure 6 . The windings are based upon the use of feed cables under 6 to $10 \mathrm{ft}$ in length.

B. Values of circuit components in figure 4.

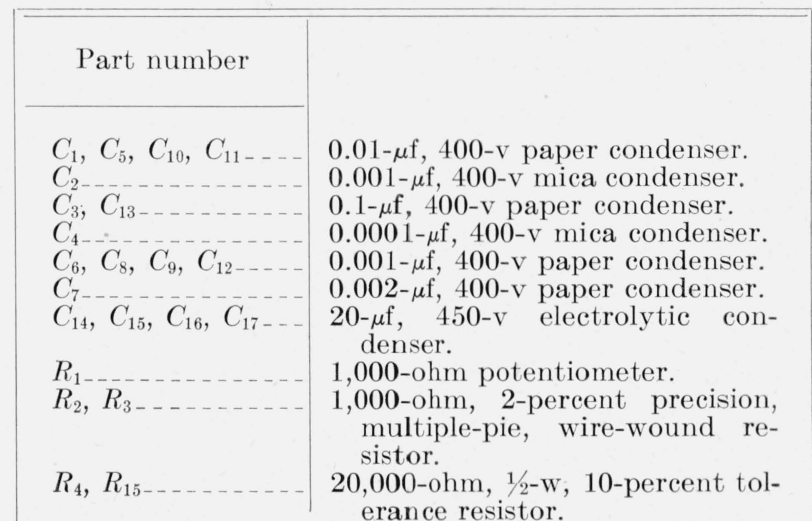




\begin{tabular}{|c|c|}
\hline Part number & \\
\hline $\begin{array}{l}R_{5} \\
R_{6} \\
R_{7}, R_{16} \ldots \\
R_{8} \\
R_{9} \\
R_{10} \\
R_{11} \\
R_{12} \\
R_{13}, R_{17} \\
R_{14} \\
R_{18} \\
R_{19} \\
S_{1} \ldots \\
V_{1} \\
V_{2} \\
V_{3} \\
V_{4} \\
V_{5} \\
V_{6} \\
\text { Vibrating relay }\end{array}$ & 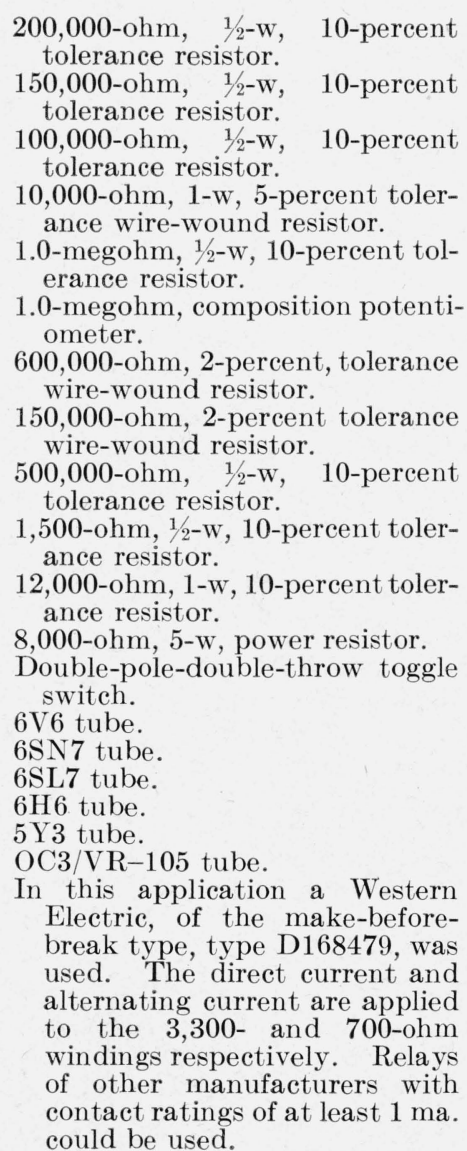 \\
\hline
\end{tabular}

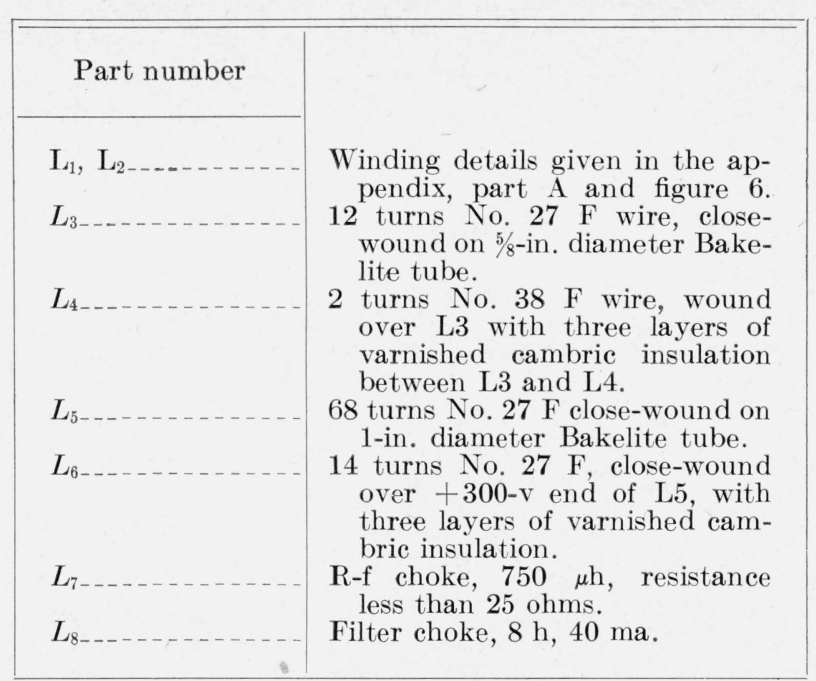

C. There may be high transient peaks coinciding with switchover of the relay. In many relays or choppers of this type, the arm may briefly touch all contacts at once, or it may have a completely open period. At the time when all contacts are joined, the combined radio-frequency voltage may not lie in the range between the individual values because of phase differences. Reversal of the polarity of either signal may be found to reduce the amplitude of the peaks. If a relay is used that has a momentary open-circuit condition, as with the break-before-make type, large transients occur because the carrier frequency amplitude then drops to zero. The effect may be eliminated by eonnecting a condenser of a few micromicrofarads between the relay arm and some source of $r-f$ voltage, such as the oscillator grid. Due to its high reactance, the condenser can only control the potential of the relay arm when it is not contacting either of the low impedance voltage sources. Proper adjustment is indicated when the transient peak is reduced to zero by establishing the open arm potential as being equal to the cancellation voltage.

Washington, June 13, 1950. 